\title{
Sensors Based on Nanoantennas: Fundamentals
}

\author{
Ricardo A. Marques Lameirinhas, João Paulo N. Torres and António Baptista
}

\section{ABSTRACT}

Nanoscience and nanotechnology are emerging fields where some phenomena were recently discovered, allowing the design of some new devices. One of these phenomena is extraordinary optical transmission EOT -, which was discovered in 1998 by Ebbesen. He reported that light can be amplified in certain conditions, due to a resonant behaviour, using metallic arrays. Even more, he associated this behaviour to surface plasmon polaritons and suggested that devices, as optical sensors, can be designed based on this phenomenon. To understand the surface plasmon polaritons theory, classical theories will be studied and compared with it. Also, the composite diffracted evanescent waves - CDEW -, model, which is not the most accurate model in comparison with the surface plasmon polaritons, will be presented, in order to cover an important topic on the theoretical foundations. After it, the application of nanoantennas as a sensor is going to be analysed. Finally, stationary simulations for a 16-slit gold array were performed using COMSOL Multiphysics and they are going to be presented in order to observe the occurrence of EOT.

Keywords: Nanoantennas · Optoelectronic devices · Sensors · Subwavelength Structures · Surface Plasmons Polaritons
Published Online: May 15, 2020

ISSN: $2684-4451$

DOI : 10.24018/ejphysics.2020.2.3.6

Ricardo A. Marques Lameirinhas* Department of Electrical and Computer Engineering, Instituto Superior Técnico, Lisbon, Portugal.

(e-mail:

ricardo.lameirinhas@tecnico.ulisboa.pt) João Paulo N. Torres

Department of Electrical and Computer Engineering, Instituto Superior Técnico, Lisbon, Portugal; Instituto de

Telecomunicações, Lisbon, Portugal.

(email: joaotorres@tecnico.ulisboa.pt) António Baptista

Department of Electrical and Computer Engineering, Instituto Superior Técnico, Lisbon, Portugal.

*Corresponding Author

\section{INTRODUCTION}

In the last decades, a huge progress in electronics and photonics allows the development of some new devices, with a large number of applications in the fields of energy, medicine, information, defence, environment and industry. Furthermore, in parallel with these technological developments, nanotechnology and nanoscience are emerging, which contributes to the miniaturization of these devices, such as medical, chemical or biological sensors, antennas for a communication system and components to photonic integrated circuits.

There is a high social and scientific recognition for those doing research or developing nanodevices and nanotechnologies. The evolution of nanotechnology as lead to the discovery of some new phenomena and to the develop of new devices and applications. However, it is dependent on several factors, such as the improvement of computational methods and the nanostructures techniques of synthesis and fabrication.

Over the years, light behaviour and its interactions with matter are inducing curiosity to the scientific community, who is trying to understand these phenomena. One of these phenomena is the Extraordinary Optical Transmission - EOT -, which was discovered by Ebbesen in 1998, who experimentally showed that a metallic array with nano holes - which is called nanoarray or nanoantenna -, can support optical transmission resonances. As it is suggested by the phenomenon designation, in certain conditions these transmission resonances can lead to light amplification and confinement - allowing the devices' miniaturisation -. Even more, this phenomenon can not be predicted by classical theories.

In the same report where Ebbesen showed, for the first time the experimental results of this phenomenon, he and his colleagues also wrote about the possibility of Surface Plasmons being the main agents of this phenomenon, pointing out some evidence of it. Although over the years some models, as the Composite Diffracted Evanescent Waves CDEW -, model have been proposed, Surface Plasmons Polaritons - SPP -, theory has always been verified as the most correct theory and until these days it is used and applied to explain this phenomenon and to find new applications.

Nowadays, EOT is used for many applications, using for example nanoantennas to exploit the aforementioned resonances. Depending on the characteristics of the material or content where the nanoantenna is placed on, the resonance behaviour is going to be different, which is the main principle to have a good sensor. This property is critical to the creation of medical, chemical or biological sensors, providing the ability to manipulate and amplify the fluorescent behaviour of some molecules. Therefore, analysis and studies about this kind of sensors, based on EOT and nanoantennas, are an attractive research theme. In this innovative research field, 
the sensors of the future are being developed for applications, in early detection of diseases or real-time checking of diseases and chemical tests.

\section{Classical Diffraction theORIES}

\section{A. Huygens' Principle and Young's Experiment}

On the XVII century, Huygens proved that every unobstructed point of a wave front behaves as a punctual source of a new spherical wave with the same characteristics of the incident one $[1,2]$. In particular, for the case of a plane wave that has normal incidence to an array of slits, each slit behaves as a punctual source. As a result of that, after the array, there are as many waves as the number of slits. However, if necessary, a more detailed analysis is possible, considering each slit as a combination of more than one punctual source $[1,2]$.

One century later, Fresnel reformulated the Huygens' principle for a curve wave front, which is used to determine the near-field regime, since near the slits, the wave is not plane. The assumption of plane waves is only valid when the target and the array are enough distanced so that, $D \gg \lambda, a$, and the wave propagation can be analysed through geometrical optics methods [1].

In 1803, Thomas Young experimentally proved the wave characteristic of light by double slit experiments [1, 2]. As illustrated on figure 1, light is normally incident on a perfect conductive plane where there are two slits of width a and spaced by $\mathrm{d}$. Also, a target is placed in parallel with the plane distanced of D.

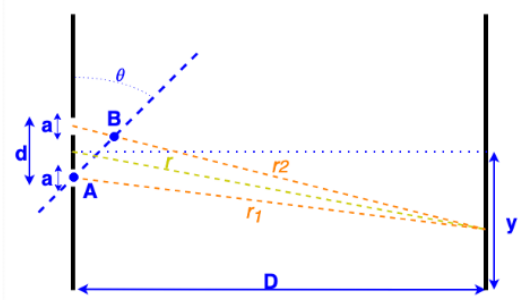

Fig. 1. Young's experiments geometry (Adapted from [1]).

Using Huygens' principle, each slit transmits one ray and since $D \gg d$, the small angle approximation is valid. Thus, $\tan (\theta)=\frac{y}{D} \approx \sin (\theta) \approx \theta$ and both rays can be considered parallels near the array, such as expressed in expression 1, where $\mathrm{x}$ is the difference between both optical paths near the slits [1].

$$
r_{2}-r_{1}=x=d \sin (\theta) \approx d
$$

If the optical path length difference of the two rays is a multiple, $m$, of $\lambda / 2$, they interfere destructively because they are in phase-opposition, expression 2 [1]. Likewise, maxima positions can be determined using expression 3 and they are related with constructive interference and in-phase waves, such that the optical path length difference of the two rays is a multiple $\mathrm{m}$ of the wavelength $\lambda[1]$.

$$
m \lambda+\frac{\lambda}{2}=d \sin (\theta) \rightarrow m \lambda+\frac{\lambda}{2} \approx d \theta \rightarrow
$$

$$
\begin{gathered}
\rightarrow y_{\text {min }} \approx(2 m+1) \frac{\lambda D}{2 d} \\
m \lambda=d \sin (\theta) \rightarrow m \lambda \approx \theta d \rightarrow y_{\text {max }} \approx \frac{m \lambda D}{d}
\end{gathered}
$$

However, it is possible to extrapolate Young's experiment to an array of $\mathrm{N}+1$ slits, such that expressions 2 and 3 can be respectively rewritten into expressions 4 and $5[1,2]$.

$$
\begin{gathered}
N m \lambda+\lambda=N d \sin (\theta) \approx N d \\
N m \lambda=N d \sin (\theta) \approx N d
\end{gathered}
$$

At this point, it is very important to distinguish what is diffraction from what is interference. The main difference between these two phenomena is the number of coherent waves in the process. Waves are classified as coherent when their frequency and direction are the same, such that their phase difference is invariant. Then, as illustrated on figure 2, for a small number of waves interacting the phenomenon is called interference otherwise, for a huge number of waves, it is called diffraction. However, as it is very difficult to say what is a huge or a small number of waves, it is not usual to distinguish these terms, since they are quite similar. Nonetheless, analysing patterns it is possible to differentiate them, since the diffraction pattern is the envelope of the interference one [1].

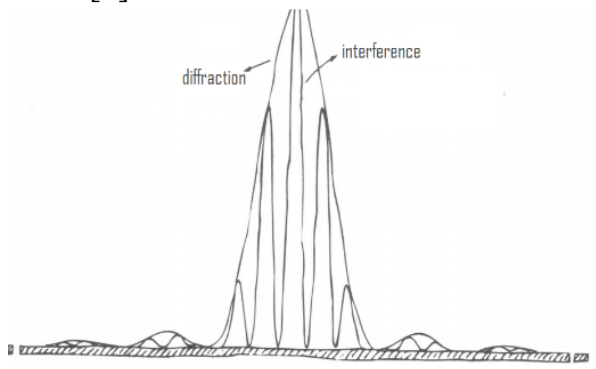

Fig. 2. Relation between diffraction and interference patterns (Adapted from [1]).

\section{B. Fraunhofer Diffraction Theory}

Assuming a plane and monochromatic wave with perpendicular incidence to a perfect conductive metal, as is illustrated in figure 3, where there is only one slit of size a on the order of the wavelength of the light -, distanced of D from a target, the ratio between the irradiance at a certain angle of diffraction $\theta$ and the main lobe one $-\theta=0^{\circ}$-, is given by Fraunhofer diffraction theory, which is mathematically presented in expression 6 , where $\lambda$ corresponds to the incident wavelength such as $k=\frac{2 \pi}{\lambda}$ and $\beta=\frac{k a}{2} \sin \theta[2,3]$. 


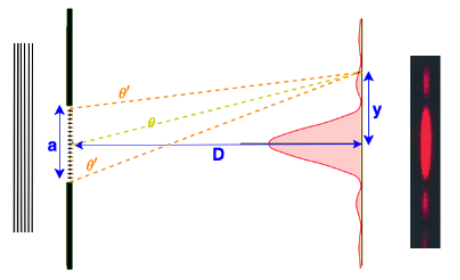

Fig. 3. Representation of Fraunhofer's ideology

According to Huygens' principle, any wave front can be replaced by a collection of punctual sources distributed uniformly over this wave front. This principle is used in Fraunhofer theory in order to divide the wave front in many sources emitting rays in phase and the maxima and minima positions along y axis can be determined using the analysis explained on section II.A., based on constructive and destructive interference. Under small angle conditions which is equivalent to state that the distance of all punctual sources to the target point is the same -, it is possible to relate $\mathrm{y}, \mathrm{D}$ and $\theta$, such as $\tan (\theta)=\frac{y}{D} \approx \sin (\theta) \approx \theta \approx \theta^{\prime}$ and thus, $\beta=\frac{a y \pi}{\lambda D}[1-3]$.

$$
\frac{I(\theta)}{I(0)}=\left(\frac{\sin (\beta)}{\beta}\right)^{2}
$$

As presented on figure 3 and verified on expression 6 , the pattern in the target is related to a sinc function. Nonetheless, under the same conditions, there is also a Fraunhofer diffraction theory of an array of $\mathrm{N}$ slits, equally spaced by $\mathrm{d}$, which is mathematically formulated as expression 7 and the relations 8 and 9 , where $I_{0}$ is the contribution of each equal slit for the overall irradiance [1]. Thus, the pattern at the target will depend on two sinc functions, one related to the size of each slit and the other associated to the distance between slits. In particular, if $\mathrm{N}=1$ and consequently $\mathrm{d}=0$, expression 7 degenerates in expression 6 .

$$
\begin{gathered}
I(\theta)=I_{0}\left(\frac{\sin (\beta)}{\beta}\right)^{2}\left(\frac{\sin (N \alpha)}{\alpha}\right)^{2} \\
=I_{0} N^{2}\left(\frac{\sin (\beta)}{\beta}\right)^{2}\left(\frac{\sin (N \alpha)}{N \alpha}\right)^{2} \\
\beta=\frac{k a}{2} \sin (\theta)=\frac{\pi a}{\lambda} \sin (\theta)=\frac{\pi a y}{\lambda D} \\
\alpha=\frac{k d}{2} \sin (\theta)=\frac{\pi d}{\lambda} \sin (\theta)=\frac{\pi d y}{\lambda D}
\end{gathered}
$$

\section{Kirchhoff Diffraction Theory}

Kirchhoff considered a plane wave incident to an opaque plane, propagating along the $\mathrm{x}$ axis, so that an aperture on this plane origin a transmitted wave determined by expression 10 [4]. Even more, Kirchhoff's theory includes as limit cases the Fresnel and Fraunhofer's theories.

$$
\nabla^{2} \bar{u}+k_{0}^{2} \bar{u}=0
$$

Using Green's theorem, it is possible to find the solution of expression 10, where the integration is based on the surface coordinates $y^{\prime}$ and z', being $x^{\prime}, y^{\prime}$ and $z^{\prime}$ the coordinates of a point $\mathbf{r}$ ' before or over the metal surface and $\varphi$ is the Green's function, for a monochromatic wave at a point $\mathbf{r}$ after the plane, given by $r^{2}=x^{2}+y^{2}+z^{2}[3,4]$. The solution is presented in expression 11 and it is based on the assumptions that $\bar{u}$ is a scalar wave and $\bar{u}$ and $\partial \bar{u} / \partial x^{\prime}$ are null after the surface, such that near the slit $\mathrm{u}=\bar{u}=\mathrm{u}_{0}$, considering $u_{0}$ as the field intensity of the incident wave, without disturbances $[3,4]$.

$$
\begin{gathered}
u(\boldsymbol{r})=\frac{1}{4 \pi} \int\left[-\frac{\partial u}{\partial x^{\prime}}\left(\boldsymbol{r}^{\prime}\right) \boldsymbol{\phi}\left(\left|\boldsymbol{r}-\boldsymbol{r}^{\prime}\right|\right)\right. \\
\left.+u\left(\boldsymbol{r}^{\prime}\right) \frac{\partial \boldsymbol{\phi}}{\partial x^{\prime}}\right] d \sigma
\end{gathered}
$$

Most of all, the previous results are the base to analyse wave propagation for an aperture dimension greater than the incident wavelength. Then, theory extension for subwavelength apertures is expressed in 12 , only valid for $k a \ll 1$ where $a$ is the aperture diameter and $\mathrm{A}$ its area $[3,4]$.

$$
u(\boldsymbol{r})=-A\left[\frac{\partial u_{0}}{\partial x^{\prime}} \boldsymbol{\phi}(\boldsymbol{r})+u_{0} \frac{\partial \boldsymbol{\phi}(\boldsymbol{r})}{\partial x}\right]
$$

\section{Bethe Diffraction Theory}

Kirchhoff's theory assumes an opaque and finite plane and consequently, boundary conditions are not satisfied at the metal surface, namely at the slit limits, such that both incident and reflected waves did not exist over the surface. However, when considering subwavelength slits, this approximation does not guarantee correct results. Thus, in 1944, Hans Albrecht Bethe amended Kirchhoff's theory, assuming a plane wave normally incident to an infinite, perfect conductive metal surface, propagating along $\mathrm{z}$ and polarized along $\mathrm{x}$ direction. A subwavelength circular hole on the metal surface is studied as a cavity upon oscillation, making three main assumptions [4]: (1) incident and reflected waves coexist over the metal surface; (2) the electromagnetic wave does not penetrate the surface, due to the infinity metal conductivity; (3) The complex electric field vector, $\overline{\boldsymbol{E}}$, is tangential to the surface and the complex magnetic field vector, $\overline{\boldsymbol{H}}$, is perpendicular to it.

Since the considered hole is circular, Bethe's theory is presented in cylindrical coordinates, considering $\mathbf{r}$ as the analysed point after the metal surface $-r^{2}=x^{2}+y^{2}+z^{2}-$, $\mathbf{r}^{\prime}$ a point before the surface $-\left(r^{\prime}\right)^{2}=\left(x^{\prime}\right)^{2}+\left(y^{\prime}\right)^{2}+\left(z^{\prime}\right)^{2}$ and $\mathbf{r}^{\prime}=\mathbf{0}$ is the source coordinates -, $a$ as the hole radius, $\kappa$ as the unity vector pointed to $\mathbf{r}$, as well as $\overline{\boldsymbol{E}}_{\mathbf{0}}$ and $\overline{\boldsymbol{H}}_{0}$ the electric and magnetic field complex vectors before the metal surface, if there is no hole [4].

Then, according to Bethe, Maxwell's equations and boundary conditions are satisfied since it is assumed an integration at large distance, i.e., far from the metal screen, $k r \gg 1$, where $\mathrm{k}$ is the wave vector given by expression 13 [4]. Thus, a punctual source is considered, 
leading to the approximation presented on expression 14 and to the Green's function of expression 15. Consequently, Maxwell's equations will degenerate on expressions 16 and $17[4]$.

$$
\begin{gathered}
k=\frac{\omega}{c}=\frac{2 \pi}{\lambda} \\
\nabla \boldsymbol{\phi}=i k \mathbf{\kappa} \boldsymbol{\begin{array} { c } 
{ \text { approximation } } \\
{ \text { linear } }
\end{array}} \boldsymbol{\phi}=\phi_{0}\left(1-i k \mathbf{\kappa} \boldsymbol{r}^{\prime}\right) \\
\phi_{0}=\phi\left(\boldsymbol{r}^{\prime}=\mathbf{0}\right)=\frac{e^{i k r}}{r} \\
\overline{\boldsymbol{E}}=\frac{1}{3 \pi} k^{2} a^{3} \phi_{0} \mathbf{\kappa} \times\left(2 \overline{\boldsymbol{H}_{\mathbf{0}}}+\overline{\boldsymbol{E}_{\mathbf{0}}} \times \mathbf{\kappa}\right) \\
\overline{\boldsymbol{H}}=-\frac{1}{3 \pi} k^{2} a^{3} \phi_{0} \mathbf{\kappa} \times\left(2 \overline{\boldsymbol{H}_{\mathbf{0}}} \times \mathbf{\kappa}-\overline{\boldsymbol{E}_{\mathbf{0}}}\right)
\end{gathered}
$$

Applying the Poynting's theorem at expressions 16 and 17 it is possible to determine the complex radiation power as presented on expression 18 for a certain direction to the point r [4]. Moreover, the total power transmitted for the hole is the result of an integration of expression 18 among all the diffracted angles - equivalent to all $\kappa$ directions -, resulting on expression 19.

$$
\begin{gathered}
\overline{\boldsymbol{S}}=\frac{c}{4 \pi} \overline{\boldsymbol{E}} \times \overline{\boldsymbol{H}}= \\
\frac{c}{36 \pi^{3}} \frac{k^{4} a^{6}}{r^{2}} \boldsymbol{\kappa}\left(2 \boldsymbol{\kappa} \times \overline{\boldsymbol{H}_{\mathbf{0}}}-\mathbf{\kappa} \times \boldsymbol{\kappa} \times \overline{\boldsymbol{E}_{\mathbf{0}}}\right)^{2} \\
\overline{\boldsymbol{S}_{\text {tot }}}=\frac{c}{27 \pi^{2}} k^{2} a^{6} \mathbf{\kappa}\left(4{\overline{\boldsymbol{H}_{\mathbf{0}}}}^{2}+{\overline{\boldsymbol{E}_{\mathbf{0}}}}^{2}\right)
\end{gathered}
$$

Therefore, the electric and magnetic field are proportional to $k^{2} a^{3}$ and, consequently, the radiation power is proportional to $k^{4} a^{6}$. However, if it is considered a subwavelength hole, such as $k a \ll 1$, the radiation power is going to be proportional to $(a / \lambda)^{4}$ [4]. Comparing Bethe's theory with Kirchhoff's one, it can be shown that Kirchhoff's intensity drop with $\lambda^{-2}$, comparing to the $\lambda^{-4}$ deduced by Bethe.

Another important conclusion took by Bethe is that the aperture can be modelled as an electric and a magnetic dipole, $\mathbf{P}$ and $\mathbf{M}$ respectively. As an electric dipole, the relation to the electromagnetic field given by 16 and 17 is presented on expressions 20 and 21 , as well as a magnetic dipole, the relation is detailed on expressions 23 and 21 [4-6].

$$
\begin{gathered}
\overline{\boldsymbol{E}}=k^{2} \phi_{0} \boldsymbol{\kappa} \times(\overline{\boldsymbol{P}} \times \mathbf{\kappa}) \\
\overline{\boldsymbol{H}}=k^{2} \phi_{0} \boldsymbol{\kappa} \times \overline{\boldsymbol{P}} \\
\overline{\boldsymbol{H}}=k^{2} \phi_{0} \boldsymbol{\kappa} \times(\overline{\boldsymbol{M}} \times \mathbf{\kappa}) \\
\overline{\boldsymbol{E}}=-k^{2} \phi_{0} \boldsymbol{\kappa}
\end{gathered}
$$

Replacing the electromagnetic field expressions 16 and 17 on expressions 20 to 23 , it is possible to deduce expressions 24 and 25, where the electric and magnetic dipoles are written in terms of the incident field and to the aperture radius.

$$
\begin{aligned}
& \overline{\boldsymbol{P}}=-\frac{1}{3 \pi} a^{3} \overline{\boldsymbol{E}_{\mathbf{0}}} \\
& \overline{\boldsymbol{M}}=-\frac{2}{3 \pi} a^{3} \overline{\boldsymbol{H}_{\mathbf{0}}}
\end{aligned}
$$

On top of that, Bethe analysed the electromagnetic field near the aperture, determining equations 26 and 27, which are only valid up to a distance of the same order than the wavelength [4-6]. However, they are presented in cartesian coordinates, that is useful to analyse boundary conditions.

$$
\begin{gathered}
\overline{\boldsymbol{E}(\boldsymbol{r})}=\left\{\begin{array}{c}
E_{x}=-\frac{4}{\pi} j k \sqrt{a^{2}-x^{2}-y^{2}} \\
E_{y}=0 \\
E_{z}=j k x
\end{array}\right. \\
\overline{\boldsymbol{H}(\boldsymbol{r})}=\left\{\begin{array}{c}
H_{x}=0 \\
H_{y}=\frac{1}{\mu_{0} c} \\
H_{z}=-\frac{4}{\mu_{0} c \pi} \frac{y}{\sqrt{a^{2}-x^{2}-y^{2}}}
\end{array}\right.
\end{gathered}
$$

Analysing expressions 26 and 27, it is possible to observe that boundary conditions are not totally satisfied, since $\overline{E_{z}} \neq 0$, due to the fact the Bethe neglected terms of the same order than $k a[5,6]$.

\section{E. Bouwkamp Diffraction Theory}

Years later, Bouwkamp proposed the rectification of Bethe's theory, in order to guarantee the satisfaction of boundary conditions $[5,6]$. Bouwkamp's approach is based on the Babinet's principle, so that he assumed a disk - instead of an aperture or a hole -, in order to obtain the magnetic currents for the disk's hole and to deduce the electromagnetic field relations presented on expressions 28 and 29 [5, 6]. Comparing these expressions with Bethe's ones, it is possible to observe that the magnetic field expression did not changed.

$$
\begin{gathered}
\overline{\boldsymbol{E}(\boldsymbol{r})}=\left\{\begin{array}{c}
E_{x}=-\frac{4 j k}{3 \pi} \frac{2 a^{2}-x^{2}-2 y^{2}}{\sqrt{a^{2}-x^{2}-y^{2}}} \\
E_{y}=-\frac{4 j k}{3 \pi} \frac{x y}{\sqrt{a^{2}-x^{2}-y^{2}}} \\
E_{z}=0
\end{array}\right. \\
\overline{\boldsymbol{H}(\boldsymbol{r})}=\left\{\begin{array}{c}
H_{x}=0 \\
H_{y}=\frac{1}{\mu_{0} c} \\
H_{z}=-\frac{4}{\mu_{0} c \pi} \frac{y}{\sqrt{a^{2}-x^{2}-y^{2}}}
\end{array}\right.
\end{gathered}
$$


Similar to Bethe, Bouwkamp determined the total radiation power transmitted by the hole, which is going to be different to the Bethe's one, because the electric field expression is different $[5,6]$.

$$
\overline{\boldsymbol{S}_{\text {tot }}}=\frac{64}{27 \pi} k^{4} a^{6} \overline{\boldsymbol{S}_{\mathbf{0}}}=\frac{64}{27 \pi} k^{4} a^{6} \frac{1}{2} c \epsilon_{0}{\overline{\boldsymbol{E}_{0}}}^{2}
$$

Even more, Bouwkamp proposed a new electric dipole formula, due to the reformulation of the electric field expression. As the magnetic field does not change, its dipole formula is maintained. Thus, electric and magnetic dipole formulas are presented on expressions 31 and 32, respectively $[5,6]$.

$$
\begin{gathered}
\overline{\boldsymbol{P}}=\frac{1}{3 \pi} a^{3} \overline{\boldsymbol{E}_{\mathbf{0}}} \\
\overline{\boldsymbol{M}}=-\frac{2}{3 \pi} a^{3} \overline{\boldsymbol{H}_{\mathbf{0}}}
\end{gathered}
$$

\section{Surface Plasmons Polaritons}

While classical theories were being proposed, in 1957 Ritchie started to study Surface Plasmons and Surface Plasmons Polaritons, later deeply studied by Raether [7-9]. A plasmon, which is considered a quasiparticle, is a quantum of a plasma oscillation - redistribution of plasma's free electrons -, just as light is the optical oscillation of photons. Therefore, plasmons are collective oscillations that can be coupled with photons, creating another quasiparticle called (plasmon) polariton. A polariton is the result of a strong coupling between electromagnetic waves and an electric or magnetic dipole. So, a surface plasmon and a surface plasmon polariton are not the same, despite the fact that they have a straight relation [7-9].

Then, surface plasmon polaritons are electromagnetic waves, propagating at the interface, between a metal/plasma and a dielectric. Assuming a dipole structure - for example an array of slits, grooves, holes, apertures or even a corrugation on the surface -, where light is incident on, the photons can excite coherent fluctuations of free electron charges at the metal boundary, creating plasmon oscillations. Then, these oscillations can couple with the incident light and consequently, polaritons are created and they will propagate at the interface [7-9]. The coupling is a resonant behaviour capable of amplifying and of concentrating light near the surface, due to polariton generation.

As presented in figure 4 , it is assumed a surface at $z=0$, so that there is metal/plasma for $\mathrm{z}<0$ and dielectric for $\mathrm{z}>0$. Thus, the non-null components of the electromagnetic field are presented on expression 33 - "+" for $z>0$ and "-" for $\mathrm{z}<0$-, where $\mathrm{m}$ is the indication of the material: 1 for the metal/plasma with a relative dielectric function given by $\epsilon_{1}$ and 2 for the dielectric characterised by a relative dielectric function of $\epsilon_{2}$ [9]. In this case, it is studied the most common and easier excitation of surface plasmon, which is due the electric field normal incidence, exciting a TM polariton [9]. However, there also the possibility of a surface plasmon excitation due to a perpendicular magnetic field where the polariton will be a TE wave.
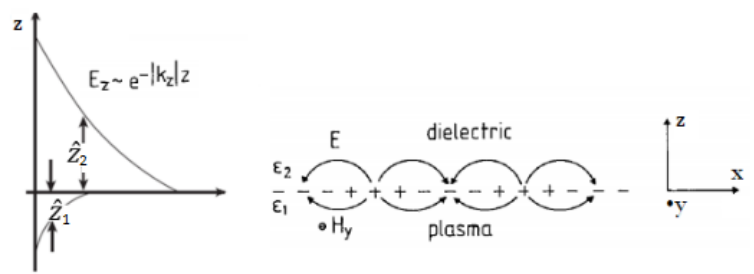

Fig. 3. Charges and electromagnetic field of surface plasmons propagation in $\mathrm{x}$ direction and $\mathrm{E}^{-} \mathrm{z}$ dependence with $\mathrm{z}$ (Adapted from [9]).

$$
\left\{\begin{array}{c}
\overline{E_{x, m}}(x, y, z, t)=E_{0} e^{j\left(\overline{k_{x}} x+\overline{k_{z, m}}|z|-\omega t\right)} \\
\overline{E_{z, m}}(x, y, z, t)= \pm \frac{\overline{k_{x}}}{\overline{k_{z, m}}} E_{0} e^{j\left(\overline{k_{x}} x+\overline{k_{z, m}}|z|-\omega t\right)} \\
\overline{H_{y, m}}(x, y, z, t)=H_{0} e^{j\left(\overline{k_{x}} x+\overline{k_{z, m}}|z|-\omega t\right)}
\end{array}\right.
$$

Thus, there are oscillations along $\mathrm{x}$ and an exponential decay over $\mathrm{z}$ direction. Another consideration that have to be done is the fact that $\overline{k_{z}}$ have different values for each material, but $\overline{k_{x}}$ will be the same for both of them, such as $\left|\overline{k_{x}}\right|=\frac{2 \pi}{\lambda_{s p}}$, where $\lambda_{s p}$ is the wavelength of the plasma oscillation. Above all, Maxwell's equations and boundary conditions - at $\mathrm{z}=0: \overline{E_{x, 1}}=\overline{E_{x, 2}}$ and $\overline{H_{y, 1}}=\overline{H_{y, 2}}$-, impose relations presented on expressions 34 , leading to the determination of $\overline{k_{x}}[3,9]$.

$$
\begin{aligned}
\boldsymbol{\nabla} \times \overline{\boldsymbol{H}}=\bar{\epsilon} \frac{\partial \overline{\boldsymbol{E}}}{\partial t} \rightarrow & \left\{\begin{array}{l}
\frac{E_{0}}{H_{0}}=-\frac{\overline{k_{z, 1}} c}{\overline{\varepsilon_{1}} \omega} \\
\frac{E_{0}}{H_{0}}=\frac{\overline{k_{z, 2}} c}{\overline{\varepsilon_{2}} \omega} \\
k^{2}=k_{x}^{2}+k_{z}^{2}
\end{array}\right. \\
& \rightarrow\left\{\begin{array}{c}
\frac{\overline{k_{z, 1}}}{\overline{\varepsilon_{1}}}+\frac{\overline{k_{z, 2}}}{\overline{\varepsilon_{2}}}=0 \\
k_{x}^{2}+k_{z, m}^{2}=\varepsilon_{m}\left(\frac{\omega}{c}\right)^{2}
\end{array}\right. \\
& \rightarrow \overline{k_{x}}=\frac{\omega}{c}\left(\frac{\overline{\varepsilon_{1}} \overline{\varepsilon_{2}}}{\overline{\varepsilon_{1}}+\overline{\varepsilon_{2}}}\right)^{1 / 2}
\end{aligned}
$$

Electromagnetic waves propagating near a metal suffer from a damping effect caused by Ohmic losses and so, it is better to consider an imaginary dielectric function $\overline{\epsilon_{1}}=\epsilon_{1}^{\prime}+j \epsilon_{1}^{\prime \prime}$ as well as, $\overline{k_{x}}=k_{x}^{\prime}+j k_{x}^{\prime \prime}$, using the relations 34. Then, it is possible to determine the formulas of $k_{x}{ }^{\prime}, k_{x}{ }^{\prime \prime}$ and $k_{z}$, as presented on expressions 35, 36 and 37 , respectively [3, 7-9].

$$
\begin{gathered}
k_{x}^{\prime}=\frac{\omega}{c}\left(\frac{\epsilon_{1}^{\prime} \epsilon_{2}}{\epsilon_{1}^{\prime}+\epsilon_{2}}\right)^{1 / 2} \\
k_{x}^{\prime \prime}=\frac{\omega}{c}\left(\frac{\epsilon_{1}^{\prime} \epsilon_{2}}{\epsilon_{1}^{\prime}+\epsilon_{2}}\right)^{3 / 2} \frac{\epsilon_{1}^{\prime \prime}}{2\left(\epsilon_{1}^{\prime}\right)^{2}} \\
k_{z, m}=\left[\epsilon_{m}\left(\frac{\omega}{c}\right)-k_{x}^{2}\right]^{1 / 2}
\end{gathered}
$$


However, it is also assumed a real $\omega$ and $\epsilon_{2}$ and also that $\left|\epsilon_{1}^{\prime}\right| \gg \epsilon_{1}^{\prime \prime}$, in order to determine independently $k_{x}^{\prime}$ and $k_{x}^{\prime \prime}$. Nonetheless, for a given incident wavelength, these expressions and assumptions lead to two important conditions for surface plasmon excitation: (1) $\epsilon_{1}^{\prime}<0$ and (2) $\left|\epsilon_{1}^{\prime}\right|>\epsilon_{2}[3,9]$. Thus, only materials with a dielectric function with negative real part and small imaginary part can support this kind of excitation [9]. The appearance of metamaterials enables the use of surface plasmons in several applications, because previously only doped semiconductors and some noble metals can fulfil these conditions. Nowadays, it is possible to design metamaterials in order to check these conditions and to reach some remarkable properties, by adjusting not only their dielectric function but also their shape, size, orientation and arrangement.

The dielectric function is dependent on the incident angular frequency $\omega$ and on the plasma bulk frequency $\omega_{p}$. Thus, the dispersion relation is presented on figure 5 , where the straight line is the light line. On its left, there is the dispersion curve of a free-space wave and on its right, the dispersion curve of a surface plasmon polariton. Furthermore, it is possible to observe that for small values of $k_{x}$ the surface plasmon polariton behaviour is similar to the light one, but as $k_{x}$ increase its behaviour tends to an asymptotic limit called surface plasmon frequency given by $\omega_{S P}=\frac{\omega_{p}}{\sqrt{1+\epsilon_{2}}}$, presenting a resonant behaviour for $\epsilon_{1}^{\prime} \rightarrow-\epsilon_{2}$, as predicted by the $\overline{k_{x}}$ expression presented on 34 .

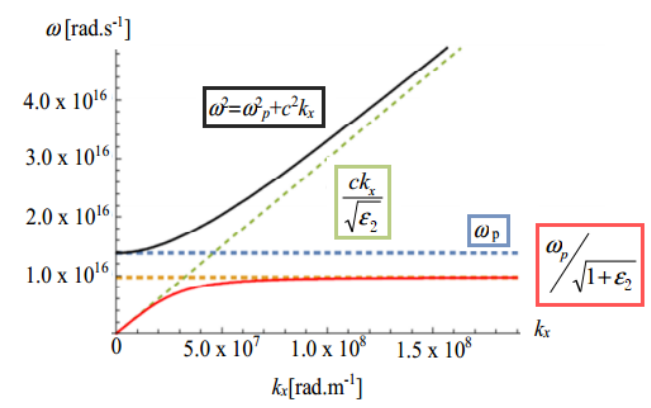

Fig. 3. Surface Plasmons dispersion relation, in this numerical example for an interface between air (dielectric) and gold (metal/plasma) (Adapted from [3]).

Therefore, as the dispersion curve is on the right side of the line one, the surface plasmon polaritons have a smaller wavelength than a free-space wave and consequently, their modes are evanescent $[3,9]$. Moreover, it is very important to evaluate the propagation distance, along $\mathrm{x}$ and $\mathrm{z}$, supported by this kind of wave. Calling penetration depth to the value of $z$ for which the electric field along this axis decays $1 / e$, it is possible to conclude that this value is given by $z=\hat{z}=\left|\overline{k_{z, m}}\right|^{-1}$, as explicitly presented on expression 38 and 39 for each material, because $E_{z} \approx e^{-\left|\overline{k_{z}}\right| z}$, as it is presented on figure $4[3,9]$.

$$
\begin{aligned}
& \widehat{z_{1}}=\frac{\lambda}{2 \pi}\left(\frac{\epsilon_{1}^{\prime}+\epsilon_{2}}{\left(\epsilon_{1}^{\prime}\right)^{2}}\right)^{1 / 2} \\
& \widehat{z_{2}}=\frac{\lambda}{2 \pi}\left(\frac{\epsilon_{1}^{\prime}+\epsilon_{2}}{\left(\epsilon_{2}\right)^{2}}\right)^{1 / 2}
\end{aligned}
$$

In the same way, it is defined the propagation length $L_{i}$ as the $\mathrm{x}$ distance where the electric field decays $1 / \mathrm{e}$. The electric field is given by $\overline{E_{x}} \approx e^{-j k_{x}^{\prime \prime} x}$, and so that $L_{i}=\left(2 k_{x}^{\prime \prime}\right)^{-1}$ or in the explicit form as presented on expression $40[3,9]$. Moreover, knowing the group velocity $v_{g}$ is possible to calculate the propagation time until the wave arrives to $x=L_{i}$ as $T_{i}=v_{g} L_{i}[9]$.

$$
L_{i}=\frac{1}{2 k_{x}^{\prime \prime}}=\frac{c}{\omega} \frac{\left(\epsilon_{1}^{\prime}\right)^{2}}{\epsilon_{1}^{\prime \prime}}\left(\frac{\epsilon_{1}^{\prime} \epsilon_{2}}{\epsilon_{1}^{\prime}+\epsilon_{2}}\right)^{2 / 3}
$$

Finally, it remains to explain how it is possible to excite surface plasmons and consequently their polaritons. One way is to use electron beams in order to exploit the electrons scattering energy, that is transferred to the plasma's bulk, to origin a wave vector parallel to the surface [9]. A surface plasmon is created with this wave vector, and since it interacts with any electromagnetic wave the polariton is generated. The other possibility is to use photons to excite a surface plasmon polariton, which is going to have the same frequency and momentum [9]. By the same reason, an incident photon can excite a free electron on the surface. As the free-space photon has more momentum comparing with a surface plasmon polariton, they cannot couple and so that, a surface plasmon is created but the polariton does not [9]. Thus, it is needed to reduce the momentum of the free-space photon, which can be done using a grating or a more complex structure with prisms. Talking about the grating - that can be an array of slits, grooves, apertures, holes or a rough surface -, if light is incident on, photons will scatter at the surface, creating a surface plasmon with the same wave vector than the parallel component of the scattered wave, but also it interacts with the scattered wave, originating the polariton [9]. In other words, momentum and energy conservation must be verified, allowing a resonant interaction between incident light and surface plasmons, creating the polaritons which are revealed as intense maxima. Also, minima are predicted by what is called Wood's anomaly [9].

\section{DRUde-LORENTZ MOdEL}

The Drude-Lorentz model relates the dielectric function to the incident wavelength or frequency. Analysing the value of $\bar{\epsilon}(\omega)$ or $\overline{\epsilon_{r}}(\omega)$, we can look for the wavelengths associated to the excitation of surface plasmons polaritons [3, 10-12].

In the XX century, the classical Drude model was combined with the quantum mechanical statistics of FermiDirac, by Arnold Sommerfeld. The DrudeSommerfeld model can describe the electric current created by an incident electromagnetic field, in a metal, just assuming the free electrons' movement [10-13]. This model also assumes a neutral metal, since the total positive ions charge is neutralised by the total negative charge of electrons [10-12]. Moreover, the ions are stopped in comparison with electrons, due to the fact that electron mass is much lower than the ions one and consequently the described current is solely influenced by electrons. Their movement is described by 
expression 41 for a point $\mathbf{r}$, where $m_{e}$ is the electron mass, $q$ is its charge, $\overline{\boldsymbol{E}}_{\mathbf{0}}$ is the complex amplitude of the incident electric field at a given angular frequency $\omega$ and the damping constant is defined as $\Gamma=\frac{v_{F}}{l}$, where $v_{F}$ is the Fermi velocity and $l$ is the average distance that one electron can travel between collisions [10-13].

$$
m_{e} \frac{\partial^{2} \boldsymbol{r}}{\partial t^{2}}+m_{e} \Gamma \frac{\partial \boldsymbol{r}}{\partial t}=q \overline{\boldsymbol{E}}_{\mathbf{0}}
$$

Using Maxwell's equations, it is possible to solve expression 41 in order to get the relative dielectric function. This solution is presented on expression 42 , where $\omega_{p}$ is the previously mentioned plasma angular frequency, which is given by $\omega_{p}=\sqrt{\frac{\tilde{n} q^{2}}{m_{e} \epsilon_{0}}}$, being $\tilde{n}$ the free electrons density $[3,10-14]$.

$$
\begin{aligned}
\bar{\epsilon}_{\text {Drude }}(\omega)=1- & \frac{\omega_{p}^{2}}{\omega^{2}+i \Gamma \omega} \\
& =1-\frac{\omega_{p}^{2}}{\omega^{2}+\Gamma^{2}}+i \frac{\Gamma \omega_{p}^{2}}{\omega\left(\omega^{2}+\Gamma^{2}\right)}
\end{aligned}
$$

This model is very accurate for infrared band applications, however its use to other spectral regions is not recommended, because there are some processes that can not be neglected. Bound electrons have to be considered to improve the model accuracy for all the spectrum, in special for visible region applications [3, 10-12]. The movement of bound electrons can be described by expression 43 , where $\mathrm{m}$ is the electron effective mass, $\gamma$ is the damping constant of radiation correlated with the bound electrons and $\alpha$ is the restitution coefficient of the potential force [3,10-12]. Solving this equation, the relative dielectric function is revealed, which is presented on expression 44 , being $\widetilde{\omega_{p}}=\sqrt{\frac{\tilde{\tilde{n}} q^{2}}{m \epsilon}}$ where $\tilde{\tilde{n}}$ is the bound electrons density and $\omega_{0}=\sqrt{\frac{\alpha}{m}}$.

$$
\begin{gathered}
m \frac{\partial^{2} \boldsymbol{r}}{\partial t^{2}}+m \gamma \frac{\partial \boldsymbol{r}}{\partial t}+\alpha \boldsymbol{r}=q \overline{\boldsymbol{E}}_{\mathbf{0}} \\
\bar{\epsilon}_{\text {bound }}(\omega)=1+\frac{{\widetilde{\omega_{p}}}^{2}\left(\omega_{0}^{2}-\omega^{2}\right)}{\left(\omega_{0}^{2}-\omega^{2}\right)^{2}+\gamma^{2} \omega^{2}} \\
+i \frac{\gamma{\widetilde{\omega_{p}}}^{2} \omega}{\left(\omega_{0}^{2}-\omega^{2}\right)^{2}+\gamma^{2} \omega^{2}}
\end{gathered}
$$

Having both, free and bound, movement described, the Drude-Lorentz model takes into account the free electrons movement and the bound electrons' harmonic oscillations. Thus, the relative dielectric constant is given by expression 45, where $\epsilon_{\infty}$ is the value of $\epsilon$ for high frequencies $[3,10-12,14]$.

$$
\begin{aligned}
\bar{\epsilon}_{D L}(\omega)=\epsilon_{\infty}- & \frac{f_{0} \omega_{p}^{2}}{\left(\omega^{2}-\omega_{0}^{2}\right)^{2}+i \omega \gamma_{0}} \\
& -\sum_{n} \frac{f_{n} \omega_{p}^{2}}{\left(\omega^{2}-\omega_{n}^{2}\right)^{2}+i \omega \gamma_{n}}
\end{aligned}
$$

\section{THE FIRST OBSERVATION}

In 1998, Ebbesen reported an unusual transmission spectrum for a nanostructure where light has normal incidence [15]. This nanostructure is a periodic hole array characterised by a periodicity constant, a hole diameter, and a thickness. Ebbesen shown huge transmission peaks on the spectrum for wavelengths larger than ten times the diameter of each hole [15]. However, these peaks are orders of magnitude greater than the predict ones, using BouwkampBethe's theory.

The absolute transmission efficiency, which is defined by Ebbesen as the ratio between the transmitted light intensity per unit area and the incident light intensity per unit area, has a value greater than 2 at the maximum, which means that more than twice the light is "transmitted as impinges directly on the holes" [15]. In addition, the experimental results reveal that the transmittivity of the array is linear proportional to the hole surface area [15]. So that, the array is considered an active element, whose characteristic behaviour can be tuned by changing the hole diameter, the periodicity of the holes, the structure thickness and the dielectric function, by changing the material [15]. Increasing the array periodicity, the maxima peaks' wavelength - spectrum position -, increased too, without dependence on the type of material, the hole diameter or the film thickness [15]. By changing the relation between the thickness and the hole diameter, the peak width will change too, as well as for different hole shapes the spectrum will be completely different [15].

Ebbesen soon reported that the main responsible agents for this remarkable phenomenon are surface plasmons, because in several tests he observed the same behaviour as when surface plasmons, excited in metallic gratings and coupled with incident light, creating polaritons [15]. Thus, extraordinary optical transmission was discovered and sooner Ebbesen stated that this phenomenon can be the base for several new optical and photonic devices [15].

\section{Composite Diffracted Evanescent WAVEs Model}

In 2004, Lezec and Thio proposed one model confronting the surface plasmon polaritons theory, named composite diffracted evanescent waves - CDEW -, in which the resonant behaviour is not due to the surface plasmon polaritons mechanism, but is due to the interference of diffracted evanescent waves [16]. They reported that the transmission enhancement factors were not of the orders of 100 to 1000 , but it should be considered a value around $7[16,17]$.

According to this model, at the slit a huge number of evanescent waves with different wave vectors values are launched. The superposition of these waves is called a composite evanescent wave, which is the sum of all diffracted inhomogeneous modes [16]. This wave propagates with a well-defined wave vector, a finite phase and an amplitude characterised by a decay with the propagation distance $[16$, 
17]. Lezec and Thio reported that their model could be used to characterise the electric field at any frequency along time and space, with or without apertures, by expression 46 , for $\mathrm{z}=0$, a wave vector magnitude equal to the source $k_{0}=\frac{2 \pi}{\lambda}$ and $\operatorname{Si}(\alpha)=\int_{0}^{\alpha} \frac{\sin (t)}{t} d t$ [16]. Although Fraunhofer theory is only valid for radiative modes $\left(0<k_{x}<k_{0}\right)$, they extended his theory for all the range $0<k_{x}<\infty$ and proceeded to a numerical integration of the weighted modes spectrum, for $x>a$, leading to expression 47 . It is possible to verify, based on this expression, that the electric field decays at $1 / x$ and it has a phase difference of $\pi / 2$ [16].

$$
\begin{gathered}
\boldsymbol{E}(x, 0)=\left\{\begin{array}{c}
-\frac{E_{0}}{x}\left[S i\left(k_{0}\left(x+\frac{a}{2}\right)\right)-S i\left(k_{0}\left(x-\frac{a}{2}\right)\right)\right],|x|>\frac{a}{2} \\
-\frac{E_{0}}{x}\left[\pi-S i\left(k_{0}\left(x+\frac{a}{2}\right)\right)+S i\left(k_{0}\left(x-\frac{a}{2}\right)\right)\right],|x|<\frac{a}{2}
\end{array}\right. \\
\boldsymbol{E}(x)=\frac{E_{0}}{\pi} \frac{a}{x} \cos \left(k_{0} x+\frac{\pi}{2}\right)
\end{gathered}
$$

This model was experimentally verified not only by Lezec and Thio, but also by Gay, in the same year [17]. While Lezec and Thio used tungsten structures, Gay built a silver one. Even though tungsten has a positive real part of the dielectric constant, they observed the occurrence of EOT, as well as Gay, however with a low enhancement factor $[16,17]$. As at least one of the EOT conditions is not verify, these experiments could be used to prove that the field amplification is not due to surface plasmon polaritons.

Two years later, in 2006, Lalanne and Hugonin developed a study based on Maxwell's equations, showing that the interaction between light and the structures as two contributions, one from the surface plasmons polaritons and other from a creeping wave [18]. Even more, they demonstrated that the polaritons are the main agents of this interaction, at least for the visible region [18]. Their report refuted the CDEW model, because they show similar results to the surface plasmon polaritons theory and huge differences in comparison to the CDEW model, having the Maxwell's equations as the initial point to their work [18]. They suggested that experimental results were spoiled by impurities and the theoretical model has the same limitations than Kirchhoff's theory [18].

\section{The Use of Nanoantennas as Sensors}

As previously referred, one way of enhancing extraordinary optical transmission is to use arrays, also called nanoantennas. As a subwavelength optical antenna, this technology enables the manipulation and control of optical radiation at micro and nano scales $[3,19]$.

The design of a nanoantenna for sensors can be divided into two different cases: one where the structure is used to improve an existing sensor or measurement system and another where the structure is itself the main agent of the measurement system.

As any electromagnetic wave can be amplified by a nanoantenna, for instance its use in X-rays or magnetic resonance imaging are fairly good examples of the first aforementioned case. Including nanoantennas on measurement system like these ones, it is not only possible to diminish the power needed per scan but also to focus radiation such that, the maximum intensity of the field will be only near the wound or illness area leading to a less intense radiation exposure on the healthy areas. Thus, nanoantennas can be used to improve the photodetection or the light emission, but also to improve the system's sensibility amplifying a certain spectral region and attenuating others [19].

However, a deep state-of-art review shows that nanoantennas are being used as sensors, rather than as an improvement to a measurement system [14, 15, 19-33]. This recent type of sensors, based on nanoantennas and EOT properties, are allowing to monitor the presence of certain molecules or cell types, for example it is quite common to being used to verify proteins. Furthermore, a sensor based on nanoantennas has been purposed to the detection of cancerous cells. There is a huge opportunity to create new sensors based on EOT, but it is needed to overcome some issues in order to design and build a sensor that is able to penetrate in the market [22, 23]. As in the majority of nanotechnologies, these issues are related to fabrication costs and reproducibility [22, $23,34]$. To reduce costs and to be able to create structures, computational methods and techniques of semiconductors and nanomaterials' synthesis must be improved. The most common techniques to produce nanoantennas are also divided into two different methods: the top-down methods such as ion beam milling or electron-beam lithography and also the bottom-up self-assembly schemes [19, 22, 23]. Furthermore, if these nanostructures are metal made, they are chemically stable, enduring long duration ultraviolet irradiation and a high temperature range [24].

Comparing sensors based on nanoantennas with other plasmonic structures its nano-size leads to the capability of miniaturising the sensor, the possibility to measure not only in vitro but also in vivo, the potential for multiplexing and to have low limits of detection [19, 22, 23, 34]. However, researchers are trying to improve some figures of merit such as sensitivity, detection limits, selectivity and dynamic range, as well as to create standalone and portable devices, with the capability of give real-time information to the user [19, 22, $23,34]$. To reach these objectives, new types of nanoantennas are being developed, as well as a completed system composed by the nanoantenna and several other devices, as internal spectrometers, angle scanners or a tunable laser $[19,22,23,34]$.

The majority of nanoantennas sensors are based on the wavelength shift of the resonance peak [20, 21]. Depending on the monitored conditions, the resonance peak position will change and consequently the intensity value too. However, the intensity peak value must be detectable in order to determine its position and to conclude about the shift value.

As previously referred, to excite surface plasmons with gratings, momentum and energy conservation must be satisfied, which is mathematically expressed by expression 48 , for displacement vectors $\widehat{e_{1}}$ and $\widehat{e_{2}}$ and, an incident angle $\theta[25,26,35]$. If it is considered a square array $-\widehat{e_{1}}=\hat{x}$ and $\widehat{e_{2}}=\hat{y}$-, with circular holes, the reciprocal array vectors are $G_{x}=G_{y}=\frac{2 \pi}{a_{0}}$, expression 49 must be satisfied, where $\mathrm{m}$ 
and $\mathrm{n}$ are the integers values associated to surface modes $[25,26,35]$. It should be noted that, in order to free-space photons $-\overline{k_{x}}-$, couple to plasmons $-\overline{k_{s p}}-$, their momentum was reduced by the gratings, such that the final momentum should be equal to $\overline{k_{x}}$ of a surface plasmon - compare expressions 49 and 34 -.

$$
\begin{gathered}
\left\{\overline{\boldsymbol{k}_{\boldsymbol{S P}}}=\overline{\boldsymbol{k}_{\boldsymbol{x}}}+m \overline{\boldsymbol{G}_{\boldsymbol{e} \mathbf{1}}}+n \overline{\boldsymbol{G}_{\boldsymbol{e} \mathbf{2}}}=k_{x}+m G_{x}+n G_{y}\right. \\
k_{x}=\frac{2 \pi}{\lambda} \sin \theta \\
k_{s p} \simeq \mathrm{R}\left\{\overline{\boldsymbol{k}_{\boldsymbol{s} p}}\right\}=\mathrm{R}\left\{\frac{\omega}{c}\left[\frac{\overline{\epsilon_{1}} \epsilon_{2}}{\overline{\epsilon_{1}}+\epsilon_{2}}\right]^{1 / 2}\right\}= \\
=\left[\left(\frac{2 \pi}{\lambda} \sin \theta+m \frac{2 \pi}{a_{0}}\right)^{2}+\left(n \frac{2 \pi}{a_{0}}\right)^{2}\right]^{1 / 2}
\end{gathered}
$$

At normal incidence $\left(\theta=0^{\circ}\right)$, the peak position, given by expression 50, can be deduced from expression $49[25,35$, 36]. In this expression, $a_{0}$ is the slit periodicity, that is linearly related to the wavelength correspondent to the maximum intensity. A similar deduction can be done for a triangular array - also called hexagonal or diagonal square centred -, resulting expression $51[25,35,36]$.

$$
\begin{aligned}
& \lambda_{\text {max }_{\text {square }}}=\mathrm{R}\left\{\left[\frac{\overline{\epsilon_{1}} \epsilon_{2}}{\overline{\epsilon_{1}}+\epsilon_{2}}\right]^{1 / 2}\right\} \times \frac{a_{0}}{|m \hat{x}+n \hat{y}|}= \\
& =a_{0}\left(m^{2}+n^{2}\right)^{-1 / 2}\left[\frac{\epsilon_{1}^{\prime} \epsilon_{2}}{\epsilon_{1}^{\prime}+\epsilon_{2}}\right]^{1 / 2} \\
& \lambda_{\text {max }_{\text {tri }}}=\mathrm{R}\left\{\left[\frac{\overline{\epsilon_{1}} \epsilon_{2}}{\overline{\epsilon_{1}}+\epsilon_{2}}\right]^{1 / 2}\right\} \times \frac{a_{0}}{\left|m \widehat{e_{1}}+n \widehat{e_{2}}\right|}= \\
& =a_{0}\left(\frac{4}{3}\left(m^{2}+m n+n^{2}\right)\right)^{-1 / 2}\left[\frac{\epsilon_{1}^{\prime} \epsilon_{2}}{\epsilon_{1}^{\prime}+\epsilon_{2}}\right]^{1 / 2}
\end{aligned}
$$

Whichever the geometry of the array and the value of $\theta$, an expression for $\lambda_{\text {max }}$ can be written as presented on expression 52 , where $\mathrm{b}$ is a parameter that depends on the array geometry $[25,35,36]$.

$$
\lambda_{\max }=\frac{a_{0}}{b}\left[\left(\frac{\epsilon_{1}^{\prime} \epsilon_{2}}{\epsilon_{1}^{\prime}+\epsilon_{2}}\right)^{1 / 2}-\sin \theta\right]
$$

Moreover, the transmission intensity peak value, for circular holes, is given by expression 53, where $\mathrm{C}$ is a constant, $\mathrm{h}$ is the hole depth and $\mathrm{a}$ is the hole diameter. Thus, it is possible to relate expressions 50 or 51 , with expression 53 , such that increasing the periodicity, the peak intensity will increase too, until saturate in a value given by $\mathrm{C}[37,38]$. In order to consider expression 53, it must be assumed that surface plasmon modes are just on one side of the nanoantenna or, if they are on both sides they should be uncoupled.

$$
I\left(h, \lambda_{p}, a\right)=C \exp \left\{-\frac{4 \pi h}{\lambda_{\max }} \sqrt{\left(\frac{\lambda_{\max }}{1.7 a}\right)^{2}-1}\right\}
$$

Also, it is possible to observe dips or minima in the transmission spectrum, that are related to the Wood's anomaly, which was reported by Wood in 1902 [7, 8, 20, 25, 35]. The wavelength value associated to the intensity minimum occurs when the diffracted light arises parallel to the metal surface, which is equivalent to say that the wave vector component perpendicular to the surface, $k_{z}$, must be considered null, resulting in expression 54. Comparing minimum and maximum expressions, it is possible to observe that the wavelength value for the maximum should be higher than the minimum one.

$$
\lambda_{\min }=a_{0} \sqrt{\epsilon_{2}}
$$

However, comparing theoretical values, expressions 50 and 51, with experimental values, the expressions are a valid first approximation, but a phase term imposed by the grating is neglected. This phase term depends on the hole shape or geometry leading to $k_{s p}=k_{x}+x G_{x}+y G_{y}+\frac{\arg (\tau)}{a}$ and to the general peak wavelength given by $\lambda_{\max }=\mathrm{R}\left\{\left[\frac{\overline{\epsilon_{1}} \epsilon_{2}}{\overline{\epsilon_{1}}+\epsilon_{2}}\right]^{1 / 2}\right\} \times \frac{a_{0}}{|x \hat{x}+y \hat{y}|+\frac{\arg (\tau)}{2 \pi}}[20,25,35,39]$. This phase term, $\arg (\tau)$, describes the effect of the hole geometry on the effective dielectric function at the surface between the dielectric and the metal. According to experimental results, the peak value is higher for holes with higher area [39]. Of course, if it is compared a circular and a square hole with a, the circular hole diameter and also a, the width of the square, the square has a greater area and then, the peak intensity will be higher.

The change of the refractive index, associate to the dielectric constant, corresponds to a shift on the peak position, or in other words, the incident wavelength that corresponds to the maximum value of light intensity changes.

The most common way to design a biomedical or biological sensor based on EOT is illustrated on figure 6, where each side of the nanoantenna is in contact with a different material. One of these materials should be solid and will be the sensors substrate - with a dielectric function $\overline{\epsilon_{3}}$-, where the metal nanoantenna is placed on the top $-\overline{\epsilon_{1}}-$. Thus, measured samples in any physical state can be placed on the top and inside the nanoantenna's holes $-\overline{\epsilon_{2}}-$. The transmission spectrum will have two different resonances peaks -, corresponding to different surface plasmon polaritons modes, because each side of the metal surface has a different dielectric constant, the solid substrate and the sample.

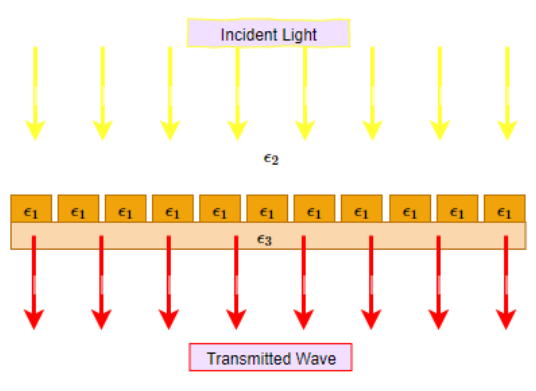

Fig. 6. Topology of the EOT peak shift sensor.

In order to test biological samples - to analyse the presence 
of a certain biological identity -, they must be placed on the nanoantenna, and after the immobilisation process, a perpendicular beam of light is incident on the samples side and the transmission spectrum is measured on the substrate side, using a spectrometer.

If the sensor's goal is to analyse a unique or a small band of wavelengths, the light emitter can be a LASER - Light Amplification by Stimulated Emission of Radiation -, otherwise a LED - Light Emitter Diode -, is a good choice. Other types of emitters, as halogen lamps, can also be used to analyse a greater band of wavelengths. The emitter should have an objective lens in order to adjust the incident power and to focus light into the sensor's active area and a polariser in order to guarantee that the incident electric field is parallel to the sensor.

Due to molecular absorption, the relative dielectric function value $\left(\overline{\epsilon_{1}}\right)$ changes and its value can be determined by a weighted average of $\bar{\epsilon}(z)$, within the extension $\hat{z}$, as presented on expression $55[20,21]$.

$$
\overline{\epsilon_{1}}=\frac{2}{\hat{z}} \int_{0}^{\infty} \bar{\epsilon}(z) e^{-\frac{2 z}{\hat{z}}} d z
$$

If it is considered an effective absorption layer thickness of $t_{d}$, the aforementioned integral is only valid until its value. Thus, the modulus of dielectric constant variation can be associated to a variation of the refractive index $\Delta \mathrm{n}$, such that $\overline{\epsilon_{r}}=(n-i k)^{2} \Leftrightarrow n=\mathrm{R}\left\{\sqrt{\overline{\epsilon_{r}}}\right\}$. Expression 56 shows the relation between a variation of the refractive index and the peak wavelength shift, where $s_{n}$ is the sensitivity of the wavelength shift to the variation of the refractive index [21, 26].

$$
\Delta \lambda_{\max }=s_{n} \Delta n\left[1-e^{-\frac{2 t_{d}}{\hat{z}}}\right]
$$

Despite the fact that the previous formulation does not take into consideration medium properties as temperature or pressure, the variation of these properties has influence on the peak position and intensity. Thus, it is also possible to design a sensor to monitor this kind of variables [28]. Its topology remains the same, as presented on figure 6 . The following formulation is done considering a region where the nanoantenna dielectric function does not vary a lot with the wavelength. This assumption allows to get a simpler formulation. Nonetheless, most devices will consider the variation of both dielectric functions - dielectric and metal -, leading to a more complex formulation.

A model for the dependence of temperature and pressure of nanoantennas transmission spectrum was proposed by Kowalski, including a set of thermodynamic equations that were related to the Lorentz-Lorentz law, where the (effective) dielectric function of the dielectric, $\epsilon_{2}$, changes [27, 28, 36], according to expression 57, where $\mathrm{T}$ is the temperature, $\mathrm{P}$ the pressure and $\mathrm{C}$ the material concentration.

According to Lorentz-Lorentz law, the refractive index and the dielectric function are related by expression 58 , being $C_{0}$ a constant reference value calculated by expression 59 , where $\rho$ is the material density and, $n_{0}$ and $\rho_{0}$ are the reference values for a certain temperature and pressure $[27,28,36]$. The material's concentrations will remain constant, for the same sensor and consequently, the last term of expression 57 is null.

$$
\begin{gathered}
d \epsilon_{2}=\left.\frac{\partial \epsilon_{2}}{\partial T}\right|_{P, C} d T+\left.\frac{\partial \epsilon_{2}}{\partial P}\right|_{T, C} d P+\left.\frac{\partial \epsilon_{2}}{\partial C}\right|_{P, T} d C \\
{\left[\frac{\epsilon_{2}-1}{\epsilon_{2}+2}\right]=\left[\frac{n_{2}^{2}-1}{n_{2}^{2}+2}\right]=C_{0} \rho} \\
C_{0}=\left[\frac{n_{2_{0}}^{2}-1}{n_{2_{0}}^{2}+2}\right]\left(\frac{1}{\rho_{0}}\right)
\end{gathered}
$$

Likewise, the dependence of the density with pressure and temperature takes into account two tabulated values: the volumetric expansion coefficient, $\beta$, and the isothermal compressibility factor, $k_{T}$. Thus, the density variation factor, $\phi=\frac{\rho}{\rho_{0}}$, expression 60 , can be replaced on expression 58 , leading to the relation expressed on $61[27,28,36]$.

$$
\begin{gathered}
\phi=\exp \left\{-\left[\beta\left(T-T_{0}\right)-k_{T}\left(P-P_{0}\right)\right]\right\} \\
n_{2}^{2}=\epsilon_{2}=\left[\frac{1+2 C_{0} \rho_{0} \phi}{1-C_{0} \rho_{0} \phi}\right]
\end{gathered}
$$

Finally, it is possible to determine the intensity peak position for the overall transmission spectrum of the nanoantenna by expression 62, where $\theta$ is the incident angle, $a_{0}$ the slit periodicity and $\mathrm{b}$ is a value related to the surface plasmon modes and array geometry $[27,28,36]$. In fact, this expression is very similar to expression 52 , by replacing $\epsilon_{2}$ for the expression that presents its variation with temperature and pressure.

$$
\lambda_{\max }=\frac{\mathrm{a}_{0}}{\mathrm{~b}}\left[\frac{\epsilon_{1}^{\prime}\left[\frac{1+2 \mathrm{C}_{0} \rho_{0} \phi}{1-\mathrm{C}_{0} \rho_{0} \phi}\right]}{\epsilon_{1}^{\prime}+\left[\frac{1+2 \mathrm{C}_{0} \rho_{0} \phi}{1-\mathrm{C}_{0} \rho_{0} \phi}\right]}-\sin (\theta)\right]
$$

\section{RESULTS OF AN EXTRAORDINARY OPTICAL TRANSMISSION SIMULATION}

In this section, EOT 2D stationary simulations of a gold nanoantenna are presented. These results were simulated using COMSOL Multiphysics, which uses a finite element method to solve modelling problems. The gold nanoantenna was simulated considering air as the surrounding dielectric device dimensions: $145 \times \lambda / 2[\mathrm{~nm}]-$. The device has an array of 16 slits of size 10 [nm] and the metal dimensions - between slits -, are $500 \times 200[\mathrm{~nm}]$. The gold is modelling according to the Drude-Lorentz model, using the parameters presented by Paul John in his article [12]. The array is away from the border in order to avoid the effects of boundary conditions.

A domain probe is placed in the dielectric in order to analyse the maximum squared ratio between the value of the maximum electric field norm and the incident one, which propagates from the bottom. The objective of these simulations is to prove that EOT occurs and also to extract the characteristic of the probe measurement in function of the 
incident wavelength, sweeping its value from $400 \mathrm{~nm}$ to $1800 \mathrm{~nm}$. The electric field on the structure is illustrated on figure 7, which is a zoom to the centre, in order to give a better perception for the EOT occurrence and of the polariton generation and propagation. This result was taken for $\lambda=1300 \mathrm{~nm}$, that is near the incident wavelength where the maximum domain field is archived - observing the sweeping results on figure 8 -. The width of the peak is so small that it is not possible to specify this wavelength without doing a sweep, due to finite precision, so that the values presented on figure 7 are not for the peak, but they are for a smaller wavelength.

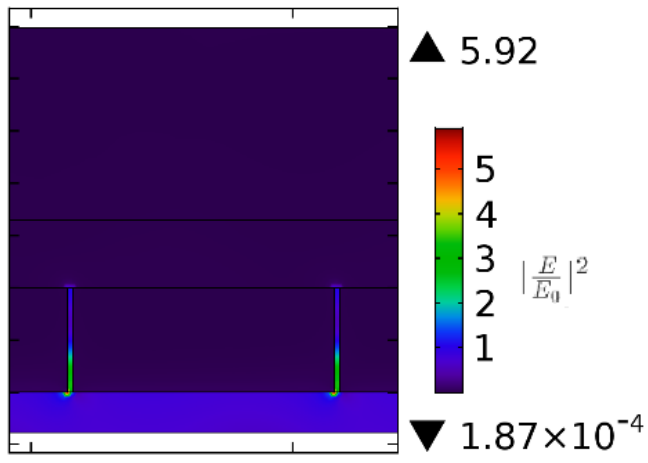

Fig. 7. Simulation of the electric field for a gold nanoantenna for $\lambda=$ $1300 \mathrm{~nm}$.
Sweeping the wavelength, the resulting characteristic is presented on figure 8 , for a total of 250 equidistant points using the domain probe. The conclusion is that the field amplification occurs for all the simulated wavelengths, because for each wavelength there is at least one domain point where the electric field norm is higher than the incident one. The values presented on figure 7 can also be verified on this characteristic.

Thus, it is possible to observe that field amplification is presented in all the spectrum, especially around 700nm and near $1300 \mathrm{~nm}$ where amplification peaks occurs, by observing figure 8 .

These results are only possible because EOT conditions are verified - especially $\epsilon_{1}^{\prime}<0$ but also $\left|\epsilon_{1}^{\prime}\right|>\epsilon_{2}$-, for all the simulated range of wavelengths, as it is possible to analyse in $\overline{\epsilon_{1}}(\omega)$ given by the fitting model computed by Paul [12]. The transmittance is not higher than one because it is a $2 \mathrm{D}$ simulation - one dimension is considered infinity -, however, it is proven that EOT occurs, despite

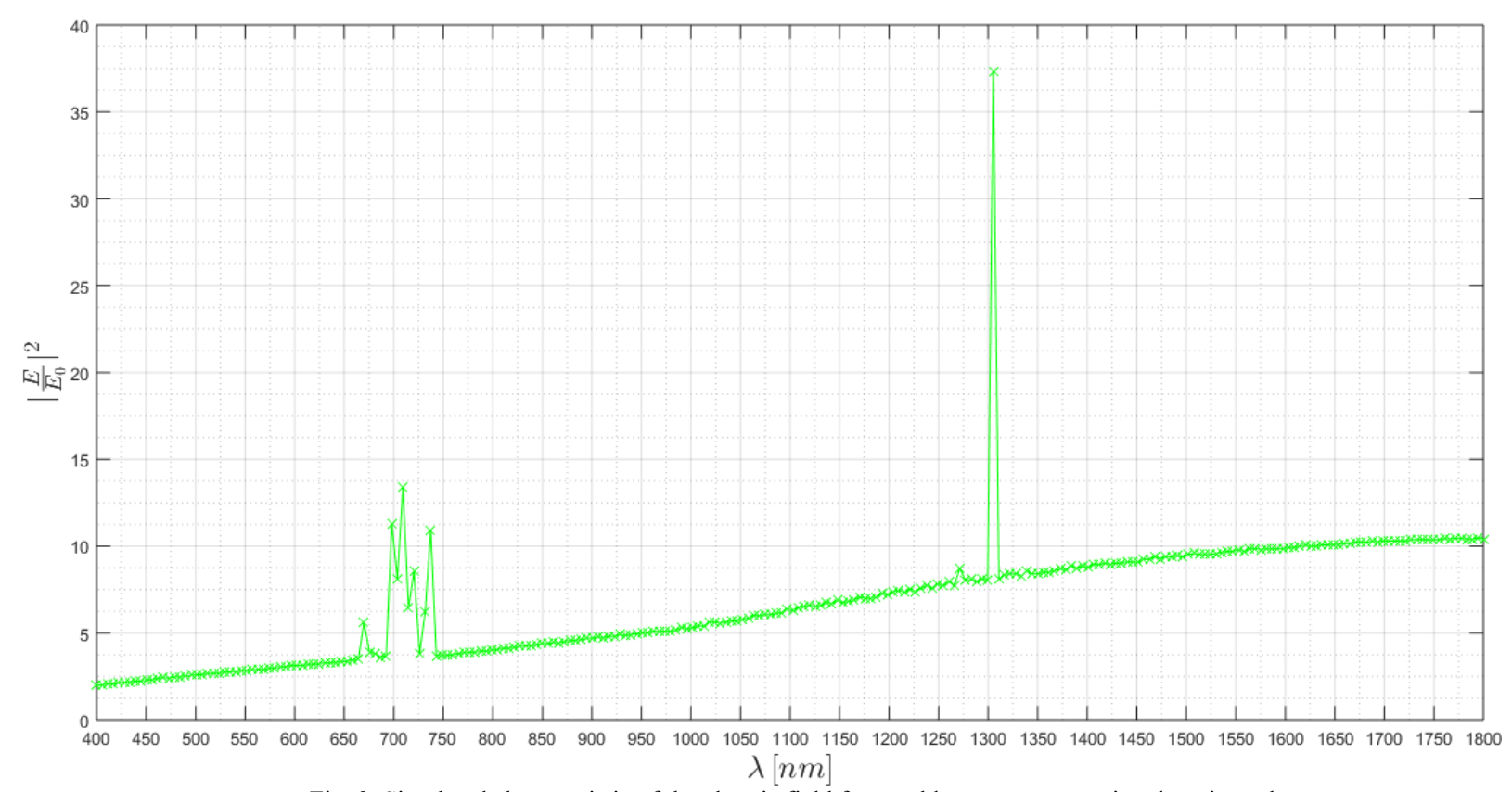

Fig. 9. Simulated characteristic of the electric field for a gold nanoantenna, using domain probe. 


\section{CONCLUSION}

To summarise, nanoantennas can be used to enhance light, what is called EOT. This phenomenon is explained as a resonant behaviour between incident light and surface plasmons. The coupling between these two kinds of waves originate surface plasmon polaritons, that are evanescent electromagnetic waves that present large differences in comparison with radiative modes. These distinct properties allow the design of new optical devices, as sensors. Sensors based on nanoantennas can be designed in order to detect or to monitor medical, chemical or biological events. Their characteristic - maximum and minimum intensity peak values and wavelength positions -, and their performance - shift sensibility, detection limits and dynamic range -, can be tuned by changing structure parameters, as the structure type and geometry, its materials and the hole shape, size or depth.

\section{ACKNOWLEDGMENT}

This work was supported in part by FCT/MCTES through national funds and in part by cofounded EU funds under Project UIDB/EEA/50008/2020.

\section{REFERENCES}

[1] Deus, J.D., et al. Princípio de Huygens. Introdução à Dísica; Escolar Editora: Lisboa, Portugal, 2014; pp. 68-88.

[2] Hecht, E. Óptica; Fundação Calouste Gulbenkian: Lisboa, Portugal, 2012; pp. 495-578.

[3] Gomes, R.D.F.R., Martins, M.J., Baptista, A. et al. Study of an Optical Antenna for Intersatellite Communications. Opt Quant Electron 2017, 49, 135.

[4] Bethe, H. A. Theory of diffraction by small holes. JPhysical review 1944, 66.7-8, 163

[5] Bouwkamp, C. J. On Bethe's Theory of Diffraction by Small Holes. Philips Research Reports 1950, 5, 321-332.

[6] Bouwkamp, C. J. Diffraction Theory. Rep. Prog. Phys 1954, 17, 35.

[7] Ritchie, R. H. Plasma losses by fast electrons in thin films. Physical review $1957,106.5,874$.

[8] Ritchie, R. H., et al. Surface-plasmon resonance effect in grating diffraction. Physical Review Letters 1968, 21.22, 1530.

[9] Raether, H. Surface plasmons on smooth surfaces. In Surface plasmons on smooth and rough surfaces and on gratings.; Springer: Heidelberg, Berlin, Germany, 1988; pp. 4-39.

[10] Sharma, N., et al. Fuchs Sondheimer-Drude Lorentz model and Drude model in the study of SPR based optical sensors: A theoretical study. Optics Communications 2015, 357, 120-126.

[11] Barchiesi, D. and Grosges, T. Fitting the optical constants of gold, silver, chromium, titanium, and aluminum in the visible bandwidth. Journal of Nanophotonics, 2014 8.1, 083097.

[12] John, P. A Drude-Time Derivative Lorentz Model for the Optical Properties of Gold. 2015.

[13] Majedi, A. H. Theoretical investigations on $\mathrm{THz}$ and optical superconductive surface plasmon interface. IEEE Transactions on Applied Superconductivity 2009, 19.3, 907-910.

[14] Tsiatmas, A. et al. Superconducting plasmonics and extraordinary transmission. Applied Physics Letters 2010, 97.11, 111106.

[15] Ebbesen, T. W., et al. Extraordinary optical transmission through subwavelength hole arrays. Nature 1998, 391, 667.

[16] Lezec, H. J. and Thio, T. Diffracted evanescent wave model for enhanced and suppressed optical transmission through subwavelength hole arrays.Optics Express 2004, 12, 3629-3651.

[17] Gay, G., et al. The optical response of nanostructured surfaces and the composite diffracted evanescent wave model. Nature Physics 2006, 2.4, 262.

[18] Lalanne, Ph., Hugonin, J. P. Interaction between optical nano-objects at metallo-dielectric interfaces. Nature Physics 2006, 2.8, 551.

[19] Novotny, L. and Niek, V. H. Antennas for light. Nature photonics 2011, 5.2, 83 .

[20] Brolo, A.G., et al. Surface Plasmon Sensor Based on the Enhanced Light Transmission Through Arrays of Nanoholes in Gold Films. Langmuir 2004, 20(12), 4813-4815.
[21] Unser, S., et al. Localized surface plasmon resonance biosensing: current challenges and approaches. Sensors 2015, 15.7, 15684-15716.

[22] Gordon, R. Extraordinary optical transmission for surface-plasmonresonance-based sensing. Nanophotonics 2008, 2, 206.

[23] Gordon, R., et al. A new generation of sensors based on extraordinary optical transmission. Accounts of chemical research 2008, 41.8, 10491057.

[24] Gu, Y., et al. Color generation via subwavelength plasmonic nanostructures. Nanoscale 2015, 7.15, 6409-6419.

[25] Thio, T., et al. Surface-plasmon-enhanced transmission through hole arrays in Cr films. JOSA B 1999, 16.10, 1743-1748.

[26] Wu, S., et al. Dielectric thickness detection sensor based on metallic nanohole arrays. The Journal of Physical Chemistry C 2011, 115.31, 15205-15209.

[27] Sen, M. A. Design and development of calorimetric biosensors using extraordinary optical transmission through nanohole arrays. 2012.

[28] Kowalski, G.J., et al. Fast Temperature Sensing Using Changes in Extraordinary Transmission Through an Array of Subwavelength Apertures. Optical Engineering 2009, 48.10, 104402.

[29] Yang, J.C., et al. Metallic Nanohole Arrays on Fluoropolymer Substrates as Small Label-Free RealTime Bioprobes. Nano Letters 2008, 8(9), 2718-2724.

[30] Hill, R. T. Plasmonic biosensors. Wiley Interdisciplinary Reviews: Nanomedicine and Nanobiotechnology 2015, 7.2, 152-168.

[31] Etezadi, D., et al. Nanoplasmonic mid-infrared biosensor for in vitro protein secondary structure detection. Light: Science \& Applications 20176.8 , e17029.

[32] Sangwan, A., et al. Increasing the Communication Distance Between Nano-Biosensing Implants and Wearable Devices. 2018 IEEE 19th International Workshop on Signal Processing Advances in Wireless Communications (SPAWC), 2018.

[33] Ji, J., et al. High-Throughput Nanohole Array Based System To Monitor Multiple Binding Events in Real Time. Analytical Chemistry 2008, 80(7), 2491-2498.

[34] Wissert, M. D., et al. Nanoengineering and characterization of gold dipole nanoantennas with enhanced integrated scattering properties. Nanotechnology 2009, 20.42, 425203.

[35] Li, J.Y., et al. Influence of hole geometry and lattice constant on extraordinary optical transmission through subwavelength hole arrays in metal films. Journal of Applied Physics 2010, 107.7, 073101.

[36] Krishnan, A., et al. Evanescently coupled resonance in surface plasmon enhanced transmission. Optics communications 2001, 200.1$6,1-7$.

[37] Degiron, A., et al. Optical Tranmission Properties of a Single Subwavelength Aperture in a Real Metals. Optics Communications 2004, 239, 61-66.

[38] Degiron, A., et al. Effects of Hole Depth on Enhanced Light Transmission Through Subwavelength Hole Arrays. Applied Physics Letters 2002, 81(23), 4327-4329.

[39] Koerkamp, K.J., et al. Strong Influence of Hole Shape on Extraordinary Transmission through Periodic Arrays of Subwavelength Holes. Physical Review 2004, 92(18), 183901. 